\title{
Antimicrobial and cytotoxic activities of fresh leaf extracts of Warburgia ugandensis
}

\author{
Z.H. MBWAMBO**, P. ERASTO, E. INNOCENT and P.J. MASIMBA \\ Institute of Traditional Medicine, Muhimbili University of Health and Allied Sciences, P.O Box 65001, \\ Dar es Salaam, Tanzania
}

\begin{abstract}
Warburgia ugandensis Sprague (Canellaceae), is widely used by Traditional Health Practitioners in Eastern and Southern Africa for treatment of diseases and conditions associated with HIV/AIDS infections. The most commonly prescribed parts of the plant are usually barks and roots. The aim of this study was to investigate whether leaves of this plant have biological activities comparable to those of barks and roots. Fresh leaves of $W$. ugandensis were freeze-dried to complete dryness while another portion of the leaves from the same collection were air-dried under shade. Ethanolic extracts from the two differently dried leaves were tested for antimicrobial activity and for brine shrimp toxicity test. Ethanolic extract from the freeze-dried leaves exhibited both antibacterial activity against standard strains of Staphylococcus aureus, Escherichia coli, Vibrio cholerae, Bacillus cereus and antifungal activity against Candida albicans and Cryptococcus neoformans. The extract from the air-dried leaves showed similar antibacterial activity against the mentioned organisms but had no antifungal activity. However, both extracts exhibited comparable cytotoxic activity (95\% CI), against brine shrimp larvae with reference to cyclophosphamide, a standard anticancer drug. Fresh leaves of W. ugandensis have shown to exhibit anticandida activity, a factor which substantiates their application as a traditional herbal remedy to complement treatment of fungal infections in HIV/AIDS patients. Further studies to establish constituents responsible for the exhibited antifungal activity are highly desirable.
\end{abstract}

Keywords: Traditional medicine, Warburgia ugandensis, Candida infections, Tanzania

\section{Introduction}

HIV / AIDS has become the world major challenging public health problem. Due to weakened immune system, HIV/AIDS patients become susceptible to opportunistic infections due to bacteria, protozoa, viruses and fungi. One among the most common fungal infection in HIV / AIDS patients is mucocutaneous candidiasis which has been reported to affect up to $90 \%$ of the patients at some point during the course of the disease (Vazquez, 2000). Oropharyngeal candidiasis (OPC) which affects the majority of persons with advanced untreated HIV infection was among the initial manifestations of HIV-induced immunodeficiency to be recognized (Gottlieb et al., 1981). Severe OPC may spread to the oesophagus and interfere with the administration of medications and adequate nutritional intake, leading to more complications in managing HIV and AIDS patients.

Candida albicans is the predominant causative agent of all forms of mucocutaneous candidiasis but other Candida species including C. glabrata, C. parapsilosis, C. tropicalis, and C. krusei, may also cause similar disease (Darouiche, 1998; Vazquez, 2000). Candidiasis can be managed well using topical, oral and systemic antifungal medication. However, the occurrence of resistance and relapse of infections are among the problems that complicate the management of candidiasis and other fungal infections in HIV/AIDS patients (Debruyne, 1997). This limitation necessitates search for new effective antifungal agents that are easily accessible and affordable by the poor majority in our local communities.

Use of traditional herbal remedies for managing human ailments has become common practice since time immemorial. The majority of people in the developing countries still rely on traditional medicines for their primary health care including HIV/AIDS management. The three species of the genus Warburgia (Canellaceae), namely, W. ugandensis Sprague, W. salutaris (Bertol.f.) Chiovand and W. stuhlmannii Engl. are widely used in traditional medicine systems of Eastern and Southern Africa for treatment of various human ailments. In South Africa the stem bark of W. salutaris is traditionally used as an expectorant, coughs, colds, sores and inflammation (Rabe \& van Staden, 2000). In East Africa dried bark of $W$. ugandensis is chewed and the juice swallowed as a remedy for stomachache, constipation, coughs, fever, toothache, muscle pains, weak joints and general body pains whereas roots are used in soup (Kokwaro, 1976). A decoction of leaves or bark is taken as a cure for malaria (Kokwaro, 1976). Among the Maasai in Kenya, the bark

\footnotetext{
* Correspondence: Zakaria H. Mbwambo; E-mail: zmbwambo@muhas.ac.tz
} 
is used for treatment of sexually transmitted diseases, stomachache, throat and chest infections, loss of appetite, malaria, diarrhoea, cough and internal wounds/ulcers, (Kiringe, 2006). In Ethiopia the stem bark of W. ugandensis is used for the treatment of tuberculosis (Wube et al., 2005). In Arusha and Manyara regions of northern Tanzania the barks and roots of $W$. ugandensis are used for treatment of HIV-related opportunistic infections, including persistent malaria, diarrhoea and flue (Kayombo et al., 2007).

Several chemical and pharmacological studies conducted to investigate extracts from the bark of Warburgia species have confirmed the presence of antifungal, antiulcer, insect antifeedant, molluscidal, antimycobacterial and antileishmanial active sesquiterpenes (Kioy et al., 1990; Lee et al. 1998; Lunde \& Kubo 2000; Wube et al., 2005; Ngure et al., 2009). Records indicate that all parts of $W$. ugandensis are edible and that leaves, bark, young shoots and fruits are used in curries while roots are used for soup (Kokwaro, 1976; Mbuya et al., 1994), suggesting that the plant is safe to human. The most commonly prescribed parts of the plant are usually barks and roots, which are harvested in the wild through de-barking and up-rooting the plant, respectively. This practice has threatened the plant to extinction. The aim of this study therefore was to investigate whether fresh leaves of $W$. ugandensis have biological activities comparable to those of barks and roots so as to justify the need for sustainable utilization of the plant and provide for its conservation.

\section{Materials and Methods}

\section{Materials}

Absolute ethanol was purchased from Fisher Scientific, UK, Ltd (Bishop Meadow Road, Loughborough, Leicestershire, LE 11 5RG, UK). Saboraud's dextrose agar (SDA) and Mueller Hinton agar were purchased from Oxoid Ltd (Basingstoke, Hampshire, England), while dimethylsulfoxide (DMSO) was purchased from Sigma (Poole, Dorset, England). Brine shrimp eggs were bought from Dohse Acquaristic, Bonn (Aus Dem Hause Dohse Acquaristik), Germany. Cyclophosphamide, a standard anticancer drug, Gentamicin susceptibility test discs $(10 \mu \mathrm{g})$ and Miconazole nitrate $(20 \mu \mathrm{g})$, were purchased from Oxoid Ltd (Basingstoke, Hampshire, England). Sea salt was prepared locally by evaporating water collected from the Indian Ocean, along the Dar es Salaam Coast.

\section{Collection, preparation and extraction of plant materials}

Fresh leaves of W. ugandensis (Canellaceae), the East African greenheart were collected from Lushoto, Tanzania in February 2008. The voucher specimen with collection No: 3420 has been deposited at the Herbarium of the Botany Department of the University of Dar es Salaam, Tanzania.

Fresh leaves of $W$. ugandensis $(500 \mathrm{~g})$ were mashed using pestle and motor and freeze-dried for $24 \mathrm{~h}$ to complete dryness. The same was powdered easily in a motor to obtain very fine aromatic green powder. A portion of this powder (200g) was extracted with $80 \%$ ethanolic aqueous by maceration, overnight, to afford extract (15.7g) labelled as extract Freeze-dried extract. Part of leaves from the same collection were air-dried to complete dryness and milled to form powdered material. A portion of the material $(200 \mathrm{~g})$ was likewise extracted with $80 \%$ ethanolic aqueous by maceration, overnight, to afford extract (12.3g) labelled as air-dried extract.

\section{Antimicrobial tests}

Antibacterial and antifungal activities were tested by the disc-diffusion method (Singh et al., 2002). Four standard bacteria, Staphylococcus aureus (NCTC 6571), Escherichia coli (NCTC 10418), Bacillus cereus (NCTC 7464), Vibrio cholerae and Proteus mirabilis (NCTC 10975) and the fungi, Candida albicans (Strain HG 392), and local strains of Cryptococcus neoformans extracted from hospitalized patients were used. Filter paper discs (Whatman No. 1; $5 \mathrm{~mm}$ diameter) were impregnated with crude extracts $(5 \mathrm{mg}$ / disc) or standard drugs (10 $\mu \mathrm{g} /$ disc gentamicin; for bacteria) and ( $20 \mu \mathrm{g} /$ disc miconazole nitrate; for fungi). The discs were overlayed on tryptone soya agar plates (for bacteria) and Saborauld's dextrose agar plates (for fungi) and incubated at $37^{\circ} \mathrm{C}$, for $24 \mathrm{~h}$. The discs were tested in triplicate, including one with a solvent blank and 3 for the standard drugs. Inhibition zones were calculated as the difference between disc diameter $(5 \mathrm{~mm})$ and the diameters of inhibition.

\section{Brine shrimp lethality test}

The brine shrimp lethality test (BST) was used to hint if the extracts may possess cytotoxic activity (Meyer et al., 1982). Assay procedures and analysis of results was done as reported earlier (Moshi \& Mbwambo, 2005). Cyclophosphamide was used as a standard anticancer test drug.

\section{Data analysis}

The mean results of brine shrimp mortality against the logarithms of concentrations were plotted using the Fig P computer programme 
(Biosoft Inc, USA), which gives also the regression equations. The latter were used to calculate $\mathrm{LC}_{16}, \mathrm{LC}_{50}$ and $\mathrm{LC}_{84}$ values. Confidence intervals $(95 \%) \mathrm{CI}$ ) were then calculated using the three results (Meyer et al., 1982). A LC Lf $_{50}$ $\geq 100 \mathrm{mg} / \mathrm{ml}$ was considered to represent an inactive extract or compound.

\section{Results}

Using the disc-diffusion method, the methanolic extracts of both freeze-dried and air-dried leaf extract of $W$. ugandensis were active against E. coli, S. aureus, V. cholera and B. cereus (Table 1). Additionally, the freeze-dried leaf extracts exhibited activity against the two fungi used, namely, C. neoformans and C. albicans, whereas the air-dried leaf extract showed no antifungal activity against the tested organisms (Table 1). toxicity, indicating other biological activity such as anti-cancer activity (Mayer et al., 1982).

It is important to note that most of the reported literature on $W$. ugandensis in traditional medicine system, has indicated use of either roots or stem barks and that the most commonly used method of harvesting the roots and stem barks from the wild has been through either uprooting and/or ring-debarking (Olila et al., 2001; Ngure et al., 2009). This practice often results in deforestation and risk to extinction of the plant species. Results from the present study provide the rationale for use of fresh leaves which grow faster and abundantly to replace barks and roots as an antifungal remedy against Candida infections. This may provide an alternative way for sustainable exploitation of $W$. ugandensis and hence contributing to conservation of this species. In that aspect, due to its valuable perception in traditional medicine system, local

Table 1: Antimicrobial test results for $W$. ugandensis leaf extracts

\begin{tabular}{lllll}
\hline Organism & $\begin{array}{l}\text { Freeze-dried } \\
\text { extract } \mathbf{8 0} \% \text { ethanol) }\end{array}$ & $\begin{array}{l}\text { leaf } \\
\text { (80\% ethanol) }\end{array}$ & $\begin{array}{l}\text { Gentamicin } \\
\text { (10ug/disc) }\end{array}$ & $\begin{array}{l}\text { Miconazole nitrate } \\
\text { (20ug/Disc) }\end{array}$ \\
\hline E. coli & $10 \mathrm{~mm}$ & $9 \mathrm{~mm}$ & $16 \mathrm{~mm}$ & - \\
S. aureus & $10 \mathrm{~mm}$ & $9 \mathrm{~mm}$ & $20 \mathrm{~mm}$ & - \\
S. typhi & - & - & $16 \mathrm{~mm}$ & - \\
S. flexineri & - & - & $16 \mathrm{~mm}$ & - \\
Ps. aeruginosa & - & - & $16 \mathrm{~mm}$ & - \\
V. cholerae & $11 \mathrm{~mm}$ & $11 \mathrm{~mm}$ & $25 \mathrm{~mm}$ & \\
P. mirabilis & - & - & $16 \mathrm{~mm}$ & \\
B. cereus & $8 \mathrm{~mm}$ & $8 \mathrm{~mm}$ & $25 \mathrm{~mm}$ & \\
C. neoformans & $9.5 \mathrm{~mm}$ & - & & $16 \mathrm{~mm}$ \\
C. albicans & $10 \mathrm{~mm}$ & - & & $17 \mathrm{~mm}$ \\
\hline
\end{tabular}

Brine shrimp lethality tests show that both extracts exhibited comparable cytotoxic activity (95\% CI), against brine shrimp larvae with reference to cyclophosphamide, a standard anticancer drug (Table 2). communities could be advised to cultivate this plant in their own yards for both medical use and perhaps for commercial purposes.

In conclusion therefore, based on the

Table 2: Brine shrimp lethality tests of $W$. ugandensis leave extracts

\begin{tabular}{lll}
\hline Extract type & LC50 $(\mu \mathrm{g} / \mathrm{ml})$ & $\mathbf{9 5} \%$ Confidence limit $(\mu \mathrm{g} / \mathrm{ml})$ \\
\hline Air-dried leaf & 24.5 & $18.5-32.5$ \\
Freeze-dried leaf & 21.6 & $14.5-32.3$ \\
Cyclophosphamide & 16.3 & $10.60-25.15$ \\
\hline
\end{tabular}

\section{Discussion}

The present study has shown that extract from fresh freeze-dried leaves of $W$. ugandensis exhibit both antibacterial and anticandida activities compared to air-dried leaves which exhibited antibacterial activity only. However, both the extracts from fresh freeze-dried and normal airdried leaves exhibited comparable brine shrimp results from this study, it is suggested that fresh leaves of $W$. ugandensis can be used as a remedy against Candida infections for managing people living with HIV. Additionally, there is a need to further investigate the fresh leaves of this plant in order to establish constituents responsible for the exhibited antifungal activity and consequently, conservation of this widely used plant in traditional medicine. 


\section{Acknowledgements}

We are grateful to Mr. Haji Selemani of the Botany Department, University of Dar es Salaam for collection and identification of the plant. This study was supported by SIDA-SAREC Research Capacity Strengthening Grant under the Muhimbili University of Health and Allied Sciences.

Received 21 March 2009

Revised 24 March 2009

Accepted 25 March 2009

\section{References}

Darouiche, R.O. (1998) Oropharyngeal and esophageal candidiasis in immunocompromised patients: treatment issues. Clinical Infectious Diseases 26, 259-274.

Debruyne, D. (1997) Clinical pharmacokinetics of fluconazole in superficial and systemic mycoses. Clinical Pharmacokinetics 33, 52-77.

Gottlieb, M.S., Schroff, R., Schanker, H.M., Weisman, J.D., Fan, P.T., Wolf, R.A. \& Saxon, A. (1981) Pneumocystis carinii pneumonia and mucosal candidiasis in previously healthy homosexual men: evidence of a new acquired cellular immunodeficiency: New England Journal of Medicine 305, 1425-1431.

Kayombo, E.J., Uiso, F. C., Mbwambo, Z.H., Mahunnah, R.L., Moshi, M.J. \& Mgonda, Y. (2007) Experience of initiating collaboration of traditional healers in managing HUV and AIDS in Tanzania. Journal of Ethnobiology and Ethnomedicine 3,6 .

Kioy, D., Gray, A.I. \& Waterman, P.G. (1990) A comparative study of the Stem-bark drimane sesquiterpenes and leaf volatile oils of $W$. ugandensis and W. stuhlimannii. Phytochemistry 29, 3535-3538.

Kiringe J.W. (2006) A survey of Traditional health remedies used by the Maasai of Southern Kajiado District, Kenya. Journal of Ethnobotany Research EApplications 4, 61-73.

Kokwaro, J.O. (1976) Medicinal Plants of East Africa, P.45. East African Literature Bureau, Nairobi, Kenya.

Lee, S.H., Lee, J.R., Lunde, C.S. \& Kubo, I. (1998) In vitro Antifungal susceptibilities of Candida albicans and other fungal pathogens to polygodial, a sesquiterpene dialdehyde. Planta Medica 65, 204-208.

Lunde, C.S. \& Kubo, I. (2000) Effect of polygodial on the mitochondrial ATPase of Saccharomyces cerevisiae. Antimicrobial Agents and Chemotherapy 44, 1943-1953.

Mbuya, L.P., Msanga, H.P., Ruffo, C.K., Birnie, A. \& Tengnäs, B. (1994) Useful trees and Shrubs for Tanzania. Identification, propagation and Management for Agricultural and Pastoral Communities. Technical Handbook No.6 pp. 510-511.

Meyer B.N.,Ferrign, R.N.,Putnam,J.E., Jacobson, L.B., Nicholas, D.E. \& McLaughlin, J.L. (1982) Brine shrimp: a convenient general bioassay for active plant constituents. Planta Medica 45, 31-34.

Moshi, M.J. \& Mbwambo, Z.H. (2005) Some pharmacological properties of extracts of Terminalia sericea roots: Journal of Ethnopharmacology 97, 43-47.

Ngure, P.K., Tonui, W.K., Ingonga, J., Mutai, CH., Kigondu, E., Ng'ang'a, Z., Rukunga, G. \& Kimutai, A. (2009) In vitro antileishmanial activity of extracts of Warburgia ugandensis (Canellaceae), a Kenyan medicinal plant. Journal of Medicinal Plants Research 3, 61-66.

Olila, D. Opunda-Asibo, J. \& Odyek, O. (2001) Bioassay-guided studies on the cytotoxic and in vitro trypanocidal activities of a sesquiterpene (Muzigadial) derived from a Ugandan medicinal plant $(W$. ugandensis). African Health Sciences 1, 1215.

Rabe, T \& J. van Staden. (2000) Isolation of an antibacterial sesquiterpenoid from Warburgia salutaris. Journal of Ethnopharmacology 73, 171-174.

Singh, B., Sahu, P.M. \& Sharma, M.K. (2002) Anti-inflammatory and antimicrobial activities of triterpenoids from Strobilanthes callosus Nees. Phytomedicine 9, 81-87.

Vazquez, J. A. (2000) Therapeutic options for the management of oropharyngeal and esophageal candidiasis in HIV/AIDS patients. HIV Clinical Trials 1, 47-59.

Wube, A.A., Bucar, F., Gibbons, S. \& Asres, K. (2005) Sesquiteroenes from Warburgia ugandensis and their antimycobacterial activity. Phytochemistry 66, 2309-2315. 